\title{
Snellen Eye Chart
}

National Cancer Institute

\section{Source}

National Cancer Institute. Snellen Eye Chart. NCI Thesaurus. Code C123467.

A diagrammatic method to test visual acuity, which utilizes a chart containing 11 lines of Sloan optotypes in varying sizes, the largest located at the top of the chart and decreasing in size toward the bottom of the chart. 